\title{
The effect of feed grains of the fatty acid composition of milk fat
}

\author{
VEIKKO KANKARE and VEIJO ANTILA
}

State Institute for Dairy Research

SF-31600 JOKIOINEN, Finland

\begin{abstract}
Finnish milk fat is relatively hard, particularly during the indoor feeding period. For reasons of both dairy technology and nutrition, however, it would be advantageous to obtain a softer fat. On the basis of the initial experiments, it would appear that the feed concentrates used, particularly their grain component, have a effect on the composition of milk fat. For this reasons, a comparative study of the effect of oats and barley on the fatty acid composition of milk fat was being carried out in the winter of 1983 .

In a comparison of barley and oats, it was observed that the iodine value of the milk in the experimental group which was fed barley was 5.1 units lower than that of the group fed oats. By itself, the feeding of barley alone was also responsible for a sharp decrease in iodine values when compared to the results obtained during the pre-experimental phase during which the cows were fed barley and oats in a ratio of $1: 1$. The softening effect of oats on milk fat appeared to be slight when the results from the experimental phase were compared to those of the pre-experimental phase.

In a comparison of the fatty acid composition of milk fat in samples from the barley and oats groups, it was found that the greatest differences were in the amounts of palmitic acid $\left(\mathrm{C}_{16}\right)$ and oleic acid $\left(\mathrm{C}_{18: 1}\right)$ present. The amount of palmitic acid in the milk fat of the barley group was $36.6 \%$, and in that of the oats group $30.2 \%$. The corresponding amounts of oleic acid were $14.7 \%$ and $22.1 \%$.

On the basis of this study, it is recommended that oats should be preferred in making up feed concentrate mixtures for milk cows.
\end{abstract}

\section{Introduction}

Finnish milk fat is relatively hard, particularly during the indoor feeding period. Despite the increased use of silage, the iodine values of milk fat have remained at precisely the same levels as prevailed in the $1960 \mathrm{~s}$ and 1970s during the indoor feeding period. For reasons of both dairy technology and nutrition, however, it would be advantageous to obtain a softer fat. The initial experiments carried out to achieve this end have shown that a few farms are already capable of producing fat during the winter that has iodine 
values on a par with those obtained during the summer. On the basis of these experiments, it would appear that the feed concentrates used, particularly their grain component, have a great effect on the composition of milk fat. For this reason, a study carried out in the late winter of 1983 to determine the effect of barley and oats on the composition of milk fat.

\section{Methods}

Experimental Feeding Scheme

A comparative study of silage and feed concentrates was being carried out at the Institute of Animal Husbandry of the Agricultural Research Centre in the late winter of 1983 , from which milk samples were obtained for the present study of the effect of oats and barley on milk fat composition. Concentrates were fed to the cows at a rate of 0.3 $\mathrm{kg} / \mathrm{kg} 4 \%$ milk. During the pre-experimental phase, oats and barley were given to the cows in the same amounts, i.e. in a ration of $1: 1$. The amounts fed the different groups during the experimental phase are shown in Table 1. The post-experimental phase connected to the present study was of necessity carried out during the period of outdoor grazing.
The experimental phase lasted c. 10 weeks. For the fat analyses, the relative milk samples were taken once a week the combined milk of four consecutive milkings. Iodine values were determined each week, and the fatty acid composition determined once during the pre-experimental phase and three times during the experimental phase.

\section{Analyses}

Fat was separated from milk by centrifuging and churning.

The iodine values were determined using the Hanus medod (see Kaufmann 1958).

Methyl esters were prepared from the fat being studied, using the method described by Christopherson and Glass (1969), Glass (1971), Christie (1973), and modified by Antila and KanKare (1983).

The composition of the fatty acids was determined in a Perkin-Elmer SIGMA 3 gas chromatograph, using an FFAP vitreous silica capillary column with a length of $25 \mathrm{~m}$ and a $\varnothing$ of $0.20 \mathrm{~mm}$. The initial temperature of the column was $70^{\circ} \mathrm{C}$, and the final temperature $220^{\circ} \mathrm{C}$. The rate of temperature rise was $7^{\circ} \mathrm{C} / \mathrm{min}$. The temperature of the injector was $250^{\circ} \mathrm{C}$ and that of the detector $280^{\circ} \mathrm{C}$. The detector was a flame ionisation detector, and hydrogen was the carrying gas.

Table 1. Scheme of Feeding Experiments

\begin{tabular}{|c|c|c|c|c|c|}
\hline \multirow{2}{*}{$\begin{array}{c}\text { Group } \\
\frac{1}{}\end{array}$} & No. of Cows & \multirow{2}{*}{$\begin{array}{c}\begin{array}{c}\text { Silage } \\
\text { ad libitum }\end{array} \\
\text { Silo } 1\end{array}$} & \multicolumn{2}{|c|}{$\begin{array}{c}\text { Concentrates } \\
- \text { controlled weekly }\end{array}$} & \multirow{2}{*}{$\begin{array}{c}\begin{array}{c}\text { Hay } \\
\mathrm{kg} / \text { day }\end{array} \\
1\end{array}$} \\
\hline & 12 & & barley & $0.3 \mathrm{~kg} / \mathrm{kg} 4 \%$ milk & \\
\hline 2 & 6 & $"$ & oats & $0.3 \mathrm{~kg} / \mathrm{kg} 4 \%$ milk & 1 \\
\hline 3 & 12 & Silo 2 & barley & $0.3 \mathrm{~kg} / \mathrm{kg} 4 \%$ milk & 1 \\
\hline 4 & 6 & $"$ & oats & $0.3 \mathrm{~kg} / \mathrm{kg} 4 \%$ milk & 1 \\
\hline 5 & 12 & Silo 3 & barley & $0.3 \mathrm{~kg} / \mathrm{kg} 4 \%$ milk & 1 \\
\hline 6 & 6 & $"$ & oats & $0.3 \mathrm{~kg} / \mathrm{kg} 4 \%$ milk & 1 \\
\hline 7 & 12 & Silo 4 & barley & $0.3 \mathrm{~kg} / \mathrm{kg} 4 \%$ milk & 1 \\
\hline 8 & 6 & $n$ & oats & $0.3 \mathrm{~kg} / \mathrm{kg} 4 \%$ milk & 1 \\
\hline
\end{tabular}




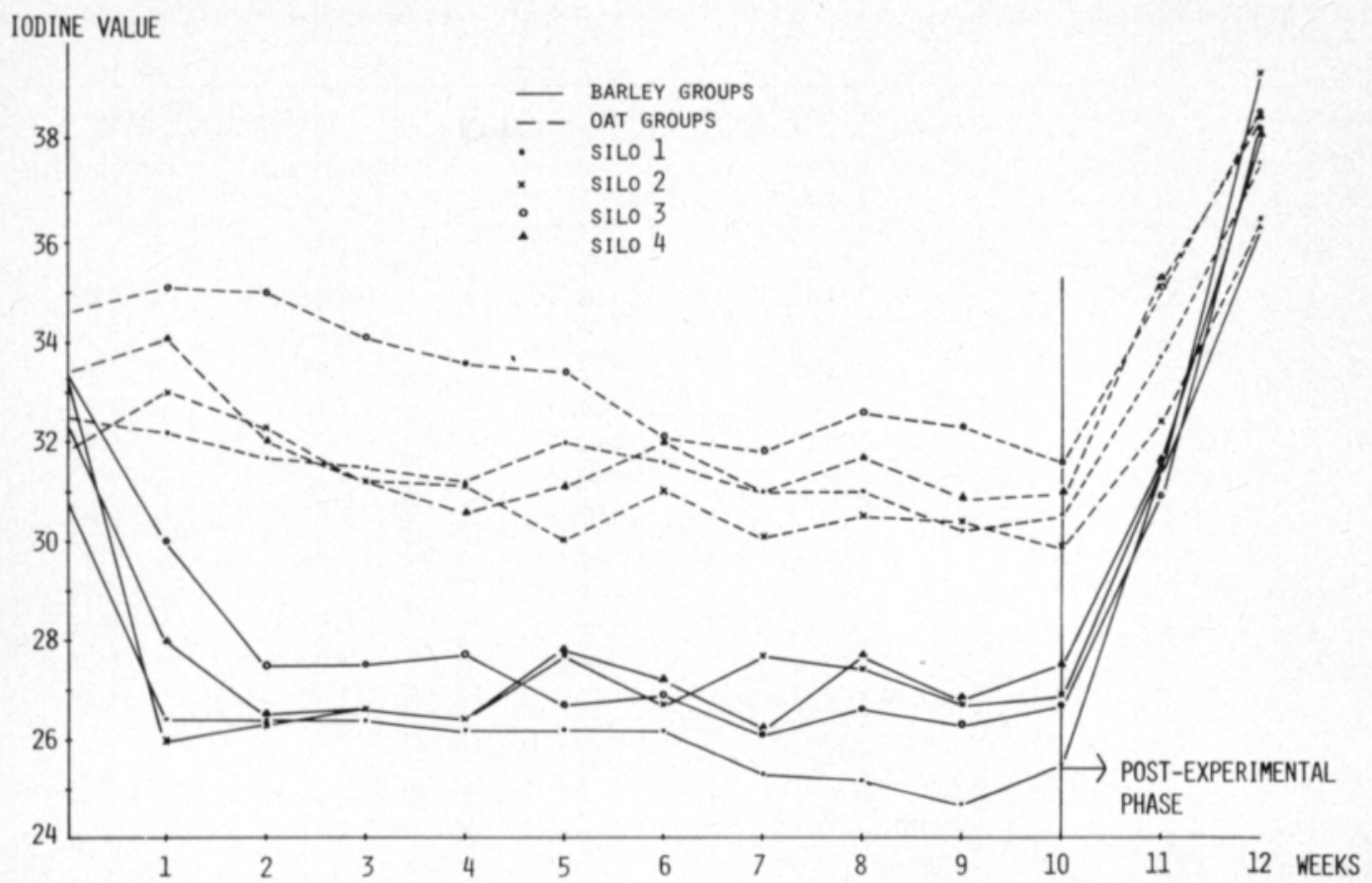

FIGURE 1. IODINE VALUES OF MILK FAT

The integrator was a Japanese Shimadzu Ltd. data processor, model C-R1B.

The fatty acids were identified utilizing standard samples (Nu-Chek-Prep 17 A, 19 A, GLC-73 and U-72-M, as well as Supelco PUFA 2), hydrated milk fat (iodine value < 0.1 ), and the literature (RENNER \& Melcher 1976, 1978 a and b, ANON. 1981).

The numbering of the fatty acids in the tables uses the same system as that based on the so-called "model chromatogram», which Kankare (1983) and Antila \& Kankare (1983) have used earlier.

\section{Results and discussion}

Iodine values

Figure 1. shows the iodine values obtained and the changes which occurred in these values at different stages of the experiment.

Feeding of barley alone caused an immediate, sharp drop in the iodine value. Feeding of oats caused a rise in the iodine value in three out of four cases. This rise was, however, not great when compared to the values obtained during feeding of the barley-oats mixture. Because of changes in the lactation period, all the iodine values dropped during the experimental phase. The average iodine value for the oats groups during the experimental phase was 31.8 , and that for the barley groups was 26.7. On this basis, it may be stated that feeding oats has a very strong hardening effect on milk fat.

In the post-experimental phase, during which the cows were moved to pasture grazing, an exceptionally sharp rise in the iodine values occurred.

Fatty acid composition

The fatty acid composition of the milk samples from the experimental groups during the pre-experimental and the experimental phases are given as average values in Tables 2 and 3.

The feeding of barley clearly increased the 
Table 2. Saturated fatty acids present in milk fat from samples taken during pre-experimental and experimental phases, \%

\begin{tabular}{|c|c|c|c|c|c|}
\hline \multirow{2}{*}{$\begin{array}{l}\text { Fatty } \\
\text { Acid } \\
\text { No. }\end{array}$} & \multirow[t]{2}{*}{ Carbon Chain } & \multicolumn{2}{|c|}{ Pre-experimental Phase } & \multicolumn{2}{|c|}{ Experimental Phase } \\
\hline & & $\begin{array}{c}\text { Groups } \\
1,3,5 \& 7\end{array}$ & $\begin{array}{c}\text { Groups } \\
2,4,6 \& 8\end{array}$ & $\begin{array}{c}\text { Barley Groups } \\
1,3,5 \& 7\end{array}$ & $\begin{array}{c}\text { Oats Groups } \\
2,4,6 \& 7\end{array}$ \\
\hline 1 & $\mathrm{C}_{4}$ & 4.35 & 4.13 & 3.63 & 3.80 \\
\hline 2 & $\mathrm{C}_{5}$ & 0.02 & 0.02 & 0.03 & 0.02 \\
\hline 3 & $\mathrm{C}_{6}$ & 2.84 & 2.75 & 2.70 & 2.56 \\
\hline 4 & $\mathrm{C}_{7}$ & 0.01 & 0.02 & 0.03 & 0.03 \\
\hline 5 & $\mathrm{C}_{8}$ & 1.70 & 1.63 & 1.72 & 1.53 \\
\hline 6 & $\mathrm{C}_{9}$ & 0.02 & 0.02 & 0.04 & 0.02 \\
\hline 7 & $\mathrm{C}_{10}$ & 3.53 & 3.43 & 4.16 & 3.34 \\
\hline 9 & $\mathrm{C}_{11}$ & 0.03 & 0.03 & 0.07 & 0.04 \\
\hline 10 & $\mathrm{C}_{12}$ & 3.58 & 3.44 & 4.62 & 3.46 \\
\hline 12 & $\mathrm{C}_{13}$ iso & 0.11 & 0.10 & 0.14 & 0.20 \\
\hline 13 & $\mathrm{C}_{13}$ & 0.07 & 0.06 & 0.10 & 0.07 \\
\hline 14 & $\mathrm{C}_{14}$ iso & 0.09 & 0.08 & 0.09 & 0.07 \\
\hline 15 & $\mathrm{C}_{14}$ & 11.95 & 11.79 & 13.36 & 11.89 \\
\hline 17 & $\mathrm{C}_{15}$ iso & 0.22 & 0.22 & 0.23 & 0.21 \\
\hline 18 & $\mathrm{C}_{15}$ anteiso & 0.35 & 0.33 & 0.33 & 0.32 \\
\hline 19 & $\mathrm{C}_{15}$ & 0.69 & 0.68 & 0.97 & 0.77 \\
\hline 22 & $\mathrm{C}_{16}$ iso & 0.23 & 0.25 & 0.20 & 0.20 \\
\hline 23 & $\mathrm{C}_{16}$ anteiso & 0.05 & 0.05 & 0.04 & 0.05 \\
\hline 24 & $\mathrm{C}_{16}$ & 30.06 & 29.84 & 36.58 & 30.21 \\
\hline 29 & $\mathrm{C}_{17}$ iso & 0.38 & 0.37 & 0.39 & 0.34 \\
\hline 30 & $\mathrm{C}_{17}$ anteiso & 0.33 & 0.34 & 0.29 & 0.29 \\
\hline 31 & $\mathrm{C}_{17}$ & 0.67 & 0.68 & 0.65 & 0.59 \\
\hline 34 & $\mathrm{C}_{18}$ iso & 0.05 & 0.05 & 0.04 & 0.04 \\
\hline 36 & $\mathrm{C}_{18}$ & 10.83 & 11.08 & 7.49 & 10.99 \\
\hline 44 & $\mathrm{C}_{19}$ & 0.06 & 0.06 & 0.06 & 0.05 \\
\hline \multirow[t]{2}{*}{49} & $\mathrm{C}_{20}$ & 0.14 & 0.15 & 0.15 & 0.15 \\
\hline & & 72.36 & 71.6 & 78.11 & 71.24 \\
\hline
\end{tabular}

amout of saturated fatty acids. The greatest differences between the feeding of barley and oats were observed, on the one hand, in the amounts of palmitic acid $\left(\mathrm{C}_{16}\right)$ present, $36.6 \%$ in the barley group and $30.2 \%$ in the oats group; and on the other hand in the amounts of oleic acid $\left(C_{18: 1}\right.$, No. 37), $14.7 \%$ in the barley and $22.1 \%$ in the oats group. 
Table 3. Unsaturated fatty acids present in milk fat from samples taken during the pre-experimental and experimental phases, \%

\begin{tabular}{|c|c|c|c|c|c|}
\hline \multirow{2}{*}{$\begin{array}{l}\text { Fatty } \\
\text { Acid } \\
\text { No. }\end{array}$} & \multirow[t]{2}{*}{ Carbon Chain } & \multicolumn{2}{|c|}{ Pre-experimental Phase } & \multicolumn{2}{|c|}{ Experimental Phase } \\
\hline & & $\begin{array}{c}\text { Groups } \\
1,3,5 \& 7\end{array}$ & $\begin{array}{c}\text { Groups } \\
2,4,6 \& 8\end{array}$ & $\begin{array}{c}\text { Barley Groups } \\
1,3,5 \& 7\end{array}$ & $\begin{array}{c}\text { Oats Groups } \\
2,4,6 \& 8\end{array}$ \\
\hline 8 & $\mathrm{C}_{10: 1}$ & 0.35 & 0.35 & 0.41 & 0.35 \\
\hline 11 & $\mathrm{C}_{12: 1}$ & 0.08 & 0.08 & 0.11 & 0.08 \\
\hline 16 & $C_{14: 1}$ & 0.91 & 0.89 & 1.12 & 0.96 \\
\hline 20 & $\mathrm{C}_{15: 1}$ & 0.03 & 0.03 & 0.04 & 0.03 \\
\hline 21 & $C_{15: 1}$ & 0.03 & 0.03 & 0.03 & 0.03 \\
\hline 21 a & $\mathrm{C}_{15: 1}$ & 0.02 & 0.02 & 0.03 & 0.02 \\
\hline 25 & $C_{16: 1}$ & 0.11 & 0.09 & 0.11 & 0.09 \\
\hline 26 & $C_{16: 1}$ & 1.30 & 1.30 & 1.47 & 1.30 \\
\hline 27 & $C_{16: 1}$ & 0.05 & 0.04 & 0.04 & 0.02 \\
\hline 28 & $C_{16: 1}$ & 0.02 & 0.02 & 0.03 & 0.02 \\
\hline 32 & $\mathrm{C}_{17: 1}$ & 0.23 & 0.25 & 0.24 & 0.25 \\
\hline 37 & $\mathrm{C}_{18: 1}$ & 20.93 & 21.38 & 14.66 & 22.1 \\
\hline 38 & $C_{18: 1}^{18.1}$ & 0.16 & 0.16 & 0.26 & 0.25 \\
\hline 39 & $C_{18: 1}$ & 0.14 & 0.14 & 0.17 & 0.18 \\
\hline 40 & $C_{18: 1}$ & 0.30 & 0.31 & 0.29 & 0.35 \\
\hline 41 & $\mathrm{C}_{18: 2}$ & 1.18 & 1.27 & 1.27 & 1.19 \\
\hline 42 & $\mathrm{C}_{18: 2}$ & 0.30 & 0.29 & 0.23 & 0.22 \\
\hline 43 & $C_{18: 2}$ & 0.03 & 0.03 & - & 0.01 \\
\hline 47 & $C_{18: 2}$ & 0.46 & 0.45 & 0.39 & 0.44 \\
\hline 45 & $C_{18: 3}$ & 0.07 & 0.07 & 0.08 & 0.08 \\
\hline 46 & $C_{18: 3}$ & 0.54 & 0.55 & 0.56 & 0.47 \\
\hline 48 & $C_{18: 4}$ & 0.04 & 0.06 & 0.08 & 0.09 \\
\hline 50 & $C_{20: 1}$ & 0.17 & 0.13 & 0.11 & 0.15 \\
\hline 51 & $C_{20: 2}$ & 0.02 & 0.05 & 0.03 & 0.04 \\
\hline 52 & $\mathrm{C}_{20: 3}$ & - & 0.02 & 0.03 & 0.03 \\
\hline 53 & $\mathrm{C}_{20: 4}$ & 0.03 & 0.03 & 0.04 & 0.03 \\
\hline \multirow[t]{2}{*}{55} & $\mathrm{C}_{20: 5}$ & 0.04 & 0.04 & 0.04 & 0.04 \\
\hline & & 27.54 & 28.04 & 21.87 & 28.7 \\
\hline
\end{tabular}

\section{References}

ANON. 1981. Identifikation of minor fedtsyrer i maelkefedt. Statens Forsøgsmejeri. Journ. nr. 2300. 34 p.

AntIla, V. \& Kankare, V. 1983. The fatty acid composition of milk lipids. Milchwissenschaft 38: 478-481.

Christopherson, S.W. \& Glass, R.L. 1969. Preparation of milk fat methyl esters by alcoholysis in an essentially nonalcoholic solution. J. Dairy Sci 52: 1289-1290.

Christie, W.W. 1973. Lipid analysis. Pergamon Press, p. 92. Oxford.

Glass, R.L. 1971. Alcoholysis saponification and the preparation of fatty acid methyl esters. Lipids 6: 919-925.

KANKARE, V. 1983. Uusia havaintoja maidon lipidikoostumuksesta. Meijeritieteellinen Aikakauskirja XLI (n:o 1) $58 \div 65$.
Kaufmann, H.P. 1958. Analyse der Fette und Fettprodukte. I. 1104 p. Berlin, Göttingen, Heidelberg.

Renner, E. \& Melcher, F. 1976. Untersuchunge über Minorfettsăuren des Milchfettes. 2. Minorfeitsăuren in Humanmilch. Milchwissenschaft 31: 193-199.

Renner, E. \& Melcher, F. 1978 a. Untersuchungen über Minorfettsăuren des Milchfettes. 3. Gesăttigte Minorfettsăuren. Milchwissenschaft 33: 281-285.

RenNer, E. \& Melcher, F. 1978 b. Untersuchungen über Minorfettsăuren des Milchfettes. 4. Ungesăttigte Minorfettsäuren Milchwissenschaft 33: 489—492.

Ms received January 27, 1984 


\section{SELOSTUS}

\section{Rehuviljan vaikutus maitorasvan rasvahappokoostumukseen}

\section{Veikko Kankare ja Veijo Antila}

Valtion maitotalouden tutkimuslaitos

Suomalainen maitorasva on erityisesti sisäruokintakaudella varsin kovaa. Huolimatta lisăăntyneestă säilörehun kảytőstă rasvan kovuutta kuvaavat jodiluvut ovat pysyneet sisäruokintakaudella täysin samalla tasolla kuin 1960- ja 1970-luvuilla. Meijeriteknologisista ja myös ravitsemuksellisista syistă olisi kuitenkin edullista saada muutetuksi rasvaa pehmeämpään suuntaan.

Alustavat kokeet osoittivat, että syötetyllă rehuviljalla on vaikutusta maitorasvan koostumukseen. Siksi Valtion maitotalouden tutkimuslaitos selvitti Maatalouden tutkimuskeskuksen kotieläinhoidon tutkimusosaston järjestämăn säilö- ja văkirehuja vertailevan tutkimuksen yhteydessă ohran ja kauran vaikutuksia maitorasvan rasvahappokoostumukseen. Koekauden aikana puolet 48 koelehmăstă sai rehuviljana pelkăstăăn ohraa ja puolet pelkästăăn kauraa.

Selvityksessä todettiin rehuviljan vaikuttavan voi- makkaasti maitorasvan koostumukseen. Ohra kovetti selvăsti tuotettua rasvaa. Ohraa syöneiden ryhmien maidon rasvan jodiluku oli 5,1 yksikkőă alempi kuin kauraa syöneiden ryhmien. Pelkän ohran syöttö alensi myős jodilukuja voimakkaasti verrattuna esikauden tuloksiin, jolloin lehmille syötettiin ohraa ja kauraa suhteessa 1:1. Kauran maitorasvaa pehmentävă vaikutus jäă văhäiseksi, kun koekauden tuloksia verrattiin esikauden tuloksiin.

Verrattaessa ohra- ja kauraryhmien rasvahappokoostumuksia toisiinsa, todettiin suurimmat erot palmitiinihapon, $\mathrm{C}_{16}$, ja öljyhapon, $\mathrm{C}_{18: 1}$ määrissă. Palmitiinihappoa ohraryhmän maitorasvassa oli $36,6 \%$ ja kauraryhmăn maitorasvassa $30,2 \%$. Vastaavat öljyhapon mäărăt olivat $14,7 \%$ ja $22,1 \%$.

Selvityksen mukaan kauraa tulisi suosia lypsylehmien văkirehuseoksissa. 